\title{
Task assignment with controlled and autonomous agents
}

\author{
Florian M. Biermann ${ }^{\mathrm{a}, *}$, Victor Naroditskiy ${ }^{\mathrm{b}}$, Maria Polukarov ${ }^{\mathrm{b}}$, Tri-Dung Nguyen ${ }^{\mathrm{c}}$, \\ Alex Rogers ${ }^{\mathrm{b}}$, Nicholas R. Jennings ${ }^{\mathrm{b}}$ \\ a International School of Economics at Tbilisi State University, Georgia \\ ${ }^{\mathrm{b}}$ Electronics and Computer Science, University of Southampton, UK \\ ' Schools of Mathematics \&' Management, University of Southampton, UK
}

\section{H I G H L I G H T S}

- We introduce an assignment problem where some agents act autonomously.

- Many real-world problems fall under this assignment model.

- Matching theory is used to model the assignment of autonomous agents to tasks.

- The problem is stated as a bi-level mathematical program.

- We transform the problem into a computationally simpler bilinear problem.

\section{A R T I C L E I N F O}

\section{Article history:}

Received 26 February 2013

Received in revised form

28 March 2014

Accepted 28 April 2014

Available online 20 June 2014

\begin{abstract}
A B S T R A C T
We analyse assignment problems in which not every agent is controlled by the central planner. The autonomous agents search for vacant tasks guided by their own preference orders over available tasks. The goal of the central planner is to maximise the total value of the assignment, taking into account the behaviour of the uncontrolled agents. Such optimisation problems arise in numerous real-world situations, ranging from organisational economics to "crowdsourcing" and disaster response. We show that the problem faced by the central planner can be transformed into a mixed integer bilevel optimisation problem. Then we demonstrate how this program can be reduced to a disjoint bilinear program, which is much more manageable computationally.
\end{abstract}

(c) 2014 Elsevier B.V. All rights reserved.

\section{Introduction}

Problems in economic theory are traditionally analysed in terms of stable outcomes (equilibria) or efficient solutions (optima). In the former case, the problem is considered in the context of the interaction of rational, self-interested, autonomous agents; in the latter, the agents are assumed to follow the instructions of the central planner who aims to optimise some objective. Of course, in realistic economic systems autonomous agents are often placed together with those controlled by the central planner, like public and private sectors jointly tackling social problems or locating economic activities. Typically, the autonomous agents will act to

\footnotetext{
* Corresponding author. Tel.: +995 322507177218.

E-mail addresses: f.biermann@iset.ge (F.M. Biermann), vn@ecs.soton.ac.uk (V. Naroditskiy), mp3@ecs.soton.ac.uk (M. Polukarov), T.D.Nguyen@soton.ac.uk (T.-D. Nguyen), acr@ecs.soton.ac.uk (A. Rogers),nrj@ecs.soton.ac.uk (N.R. Jennings).
}

obtain their own individual goals, and the problem of the central planner is to coordinate the controlled agents so as to optimise the overall performance of the system, while taking into account the behaviour of self-motivated participants.

The present paper investigates a particular "semi-autonomous" scenario of this kind, namely assignment problems in which some of the players are autonomous and face private incentives to solve certain tasks. Instead of submitting to the planner's will, these agents strive to obtain the task that rates most highly according to their own preference rankings.

The contribution of this paper is both conceptual and technical. On the conceptual level we introduce a model to handle selfish behaviour in assignment scenarios. We call this variation of the assignment problem the Semi-Autonomous Assignment Problem (SAAP). When all agents are fully controlled by the $\mathrm{CP}$, the SAAP turns into a classical assignment problem.

In our model, the autonomous agents, amended to the classical assignment problem, are assumed to have ordinal preferences over the available tasks. This arguably increases the robustness 
and applicability of our model. We neither require the central planner to form a belief about cardinal utility functions of the autonomous agents, nor do we assume the autonomous agents to be von Neumann-Morgenstern expected utility maximisers. Likewise, adopting ordinal preferences allows us to directly utilise results from a branch of game theory, usually called matching theory, which originated with the seminal paper of Gale and Shapley (1962). From the start, matching theory evolved without drawing on the theory of expected utility.

Part of our technical contribution is to show that the optimal solution of an SAAP from the point of view of the central planner corresponds to a stable matching (Gale and Shapley, 1962) in a particular marriage market ${ }^{1}$ formed by autonomous agents and tasks. In this market, the preferences of the autonomous agents are their rankings over tasks, while the values of the "assignment matrix" determine the preferences of the tasks. By assigning the controlled agents, the central planner can block some tasks and in this way essentially determine the market in which the stable matching is formed. The seemingly strong assumption that the central planner moves first does not affect the generality of our model. Making use of a result from matching literature, in Section 3 we argue that the outcome will be the same if the central planner does not move first. This is true as long as the central planner has the prerogative to assign his controlled agents to tasks even if they were already taken by autonomous agents (who in that case become unassigned again).

As we show, the optimisation problem faced by the central planner can be represented as a mixed integer bilevel optimisation problem-a hierarchical program where the set of constraints contains a parametric optimisation problem. Solving bilevel programs is difficult in general, and known algorithms would deal with only extremely small problem instances. We then show how to reduce this bilevel program to a disjoint bilinear program, using the special structure of the SAAP. A disjoint bilinear program is much more manageable computationally, as it only involves a single minimisation problem instead of a minimax problem.

The rest of the paper is organised as follows. Section 2 motivates our work by describing several real-life situations which resemble SAAPs. The model is then formally defined in Section 3. In Section 4 we present our main results-the transformation of the SAAP to a mixed integer bilevel optimisation problem and the reduction of that problem to a disjoint bilinear program. We conclude in Section 5 with directions for future work.

\section{Real-world examples}

Semi-autonomous assignment problems arise naturally in the context of location of economic activities. In Koopmans and Beckmann (1957), for example, the authors discuss the assignment problem in the context of choosing locations for industrial plants under the standard assumption that the central planner is responsible for choosing the location for all of the plants. However, in reality such tasks are typically divided between the public and the private sectors, where private businesses strive to maximise their own profits and the government is concerned with the overall welfare of the society. Note also that state institutions often have the priority over private entrepreneurs in making their choices, consistent with the assumptions of our model.

As another example, consider private-public partnerships (PPP), where the public party, which usually supervises the complete project, intends to advance some public goal. In contrast, the participating private parties are primarily interested in their own profits. This poses an obstacle for assigning tasks in a

\footnotetext{
1 Technically, a marriage market is a one-to-one two-sided matching problem.
}

globally optimal way. Companies will try to avoid those tasks which are unprofitable and difficult, trying instead to obtain subprojects promising high profits at low risk. A typical example is the provision of health care through hospitals and doctors, which is facilitated through private-public partnerships in many countries. ${ }^{2}$ The payment agreements between the government and the private partners usually do not reimburse a hospital or doctor for exactly those costs associated with a specific patient. As a result, patients (="tasks") yield different profit opportunities. Although hospitals/doctors (="agents") participating in a PPP are not formally entitled to pick the profitable patients and reject the others, there may be informal ways to deter unprofitable patients. ${ }^{3}$ The model presented in this paper can help to design policies which take the selfish behaviour of private contractors into account.

In the internet economy, many crowdsourcing systems (see, e.g., Benkler, 2006, Brabham, 2008 and Howe, 2008) can be modelled as SAAPs. In a crowdsourcing system, tasks which cannot satisfactorily be solved without human expertise are assigned to a group of more or less anonymous amateur problem solvers (the "crowd"). Yet companies making use of crowdsourcing do not have to totally rely on the crowd. For some of the tasks or even for all of them, they can engage professional problem solvers. These belong to their own personnel or a contractor's personnel who cannot reject tasks assigned to them. In contrast, crowd members can freely choose which tasks to work on, and they are probably not indifferent between all tasks. Hence, the firm has to find an optimal way of distributing its tasks between professional and amateur problem solvers.

Disaster response situations, providing prominent examples of crowdsourcing, can also be analysed with our model. Consider a disaster relief situation where professional disaster responders coordinated by the government are assisted by local residents and disaster survivors. The government has neither the communication capabilities nor the authority to tell local participants what to do. However, local participants are very helpful and their efforts should not be ignored. Assuming the government can estimate the preferences of local participants (e.g., they visit sites in order of distance from their home), our work provides a more effective way for the government to assign professional disaster responders.

Finally, autonomous task choice can even be observed in military organisations, which are famous for their strict adherence to the principle of obeying orders. ${ }^{4}$ If solving critical tasks is "prestigious" in some sense, there may be an incentive for military officers to unilaterally go for those critical tasks, disregarding the assignment the central planner would prefer. In military history it regularly occurred that ambitious commanders tried to gain fame by acting more bravely or by taking greater risks than desired by the central command. An outstanding example is the celebrated Danish naval officer Peter Jansen Wessel (1691-1720),

\footnotetext{
2 For an overview of private-public partnerships in the health sector, see Nikolic and Maikisch (2006).

3 By entering "hospital turns away" or a similar phrase into an internet search engine, one gets plenty of media reports about exactly this issue. For example, UK dentists, working for the National Health Service, arguably behaved in such a way (see Templeton, 2007). Reports about hospitals being reluctant to examine patients with X-ray or brain scans may straightforwardly be interpreted as avoidance of unprofitable tasks.

4 Situations resembling SAAPs can be found not only within military organisations. The 2011 war in Libya was fought by a coalition of NATO and loosely organised rebel troops, cooperating in their efforts to overthrow the regime of dictator Muammar Gaddafi. While the NATO forces were totally coordinated, it was arguably difficult to coordinate the actions of the rebels, who were untrained, unprofessional, and lacked command chains. Consequently, the NATO, as the central planner of the SAAP, had to anticipate the prospective actions of the rebels when making its decisions on air strikes. Information about the rebels' next steps was provided by socalled liaison officers (NATO representatives assigned to the rebel units).
} 
called Tordenskjold (Danish for "thunder shield"). He constantly strived for the most prestigious tasks in the Great Northern War (1700-1721), thereby notoriously disobeying orders. ${ }^{5}$ His confrontation with the Swedish fleet in the Battle of Dynekilen (1716) in which his 7 ships captured 31 Swedish ships and destroyed another 13 , was not backed by orders of the admiralty. ${ }^{6}$ Wessel's anarchistic conduct evoked considerable criticism in the Danish admiralty, eventually leading to a trial at a courtmartial. Yet he was acquitted and even made an admiral later. ${ }^{7}$ His disobedience yielded huge personal prestige, as can be seen from the fact that Wessel is praised in the national anthems of both Denmark and Norway (the country he originated from).

\section{The model}

Before formally defining our model, we recall the definition of a classical Assignment Problem (AP). An AP is defined by a triple $(A, T, v)$, where $A$ is a set of agents, $T$ is a set of tasks, and $v$ is an evaluation function which maps $A \times T$ into $\mathbb{R}_{+} \cup\{0\}$. The problem is to find an assignment (or, matching) of agents to tasks for which the sum of the values of pairs matched is maximised. Formally, an assignment $\mu$ is a subset of $A \times T$ such that no two distinct pairs in $\mu$ share a player or a task, that is:

$(a, t),(\hat{a}, \hat{t}) \in \mu:(a, t) \neq(\hat{a}, \hat{t}) \Rightarrow a \neq \hat{a} \wedge t \neq \hat{t}$.

The objective of the central planner is then given by

$\max _{\mu \in \mu} \sum_{(a, t) \in \mu} v(a, t)$

with $\mu$ being the set of all assignments which can be formed from the set $A \times T$.

We now generalise the AP model to what we call the SemiAutonomous Assignment Problem (SAAP). An SAAP is defined by a tuple

$\left(C \cup F, T, v, \succ_{\boldsymbol{F}}\right)$,

where $C$ and $F$ are two disjoint sets, and we set $A:=C \cup F$. As before, we refer to the elements of $A$ as agents, while the members of $C$ are termed coordinated (or, controlled), and the members of $F$ are referred to as free (or, autonomous). The function $v$ is defined as before, and $\succ_{\boldsymbol{F}}$ is a strict ${ }^{8}$ preference profile which contains for each free agent $f \in F$ a linear preference order $\succ_{f}$ defined over $T$. Given this, the central planner of an SAAP aims to find

$\max _{\mu \in \mu^{\text {SAAP }}} \sum_{(a, t) \in \mu} v(a, t)$,

where $\boldsymbol{\mu}^{\text {SAAP }}$ is the set of SAAP-feasible assignments defined later. First we specify the behaviour of the free agents.

We now specify a search process such that keeping to this process is a (weakly) dominant strategy for each free agent.

After the coordinated agents were assigned to tasks by the central planner, each free agent $f$ approaches his most preferred task $t:=\max _{\succ f} T$. If $f$ finds $t$ to be vacant, $f$ takes over $t$. If $f$ finds that a coordinated player already occupies $t, f$ proceeds to the task which is second according to the preferences $\succ_{f}$, namely

\footnotetext{
5 For an account of his deeds, see Chapter 1 ("A Knight Errant of the Seas") in Riis (2007).

6 "He could not go back and ask for permission, and one may shrewdly guess that he did not want to, for it would certainly have been refused." (Riis, 2007, p. 10).

7 Cf. Riis (2007, pp. 6 and 9).

8 The assumption of strict preferences is common in matching literature (e.g., see Chapter 2 in Roth and Sotomayor, 1990). In deriving our results, we make use of one of the standard results in matching theory (Corollary 2.14, p. 33, in Roth and Sotomayor, 1990) which holds only for strict preferences.
}

$t^{\prime}:=\max _{\succ f} T \backslash\{t\}$. Again, $f$ checks the availability of $t^{\prime}$ and either takes it or continues with the subsequent item in its priority list. If there are no tasks left on $f$ 's priority list which were not yet approached, $f$ stays idle. If $k \geq 2$ free agents $f_{1}, \ldots, f_{k}$ approach the same task $t$, we assume that the agent best at performing the task, i.e.

$\arg \max v(a, t)$

$a \in\left\{f_{1}, \ldots, f_{k}\right\}$

keeps to $t$, while the other free agents continue the search process. This is a realistic assumption for scenarios in which free players, though being uncoordinated, have an interest in a high-valued solution of the problem (like in the disaster response application outlined in Section 2). We make the restriction that $v(a, t) \neq$ $v\left(a^{\prime}, t\right)$ if $a \neq a^{\prime}{ }^{9}$ With these assumptions, the central planner affects the outcome of the search process because tasks assigned to the controlled agents are unavailable to the free agents.

If the behaviour of the free agents is modelled in this way, the search process coincides with the Deferred Acceptance Algorithm of Gale and Shapley (1962) with men proposing, where:

- The free agents in $F$ are the men and the tasks in $T$ are the women.

- The men's preferences are given by $\succ_{\boldsymbol{F}}$.

- The women's preferences are given by the valuation function $v$, i.e. for each $t \in T$ we have

$$
f \succ_{t} f^{\prime} \Leftrightarrow v(f, t)>v\left(f^{\prime}, t\right) \text {. }
$$

- Some tasks are blocked, namely those that are occupied by controlled agents.

We call this procedure the Deferred Acceptance Algorithm with Blocked Tasks (DAB).

The Deferred Acceptance Algorithm of Gale and Shapley (1962) constructs a stable matching in a marriage market. A marriage market is defined as a triple $(M, W, \succ)$, where $M$ is the set of "men" and $W$ is the set of "women". A preference profile $\succ$ maps each $m \in$ $M$ into a linear preference order defined over $W \cup\{m\}$, and each $w \in W$ into a linear preference order defined over $M \cup\{w\} .{ }^{10,11}$

From the fact that the deferred acceptance algorithm is finite and produces a unique output (Gale and Shapley, 1962), it follows that the DAB search process is finite and produces a unique output.

It is a dominant strategy equilibrium for each agent from the male side to reveal its preferences truthfully (see Theorem 5 in Roth, 1982). In our model, revealing preferences is done via the order in which tasks are approached. Since our search process specifies that tasks are approached in the order given by agent's preferences and since our search process coincides with the deferred acceptance algorithm, it means that straightforwardly following their preferences is a dominant strategy for the agents, i.e. the free agents cannot improve their outcome by changing the order in which they approach tasks.

McVitie and Wilson (1971) modified the original algorithm of Gale and Shapley (1962) so as to let men propose to women in a random sequence (in Gale and Shapley, 1962, the men propose simultaneously at each stage). They proved that the matching resulting from their algorithm is identical to the one generated by the standard deferred acceptance algorithm. This finding of

\footnotetext{
9 In the marriage market we define, this restriction will ensure that "preferences of tasks" are strict. This assumption is needed for our results (see also Footnote 8 above).

10 It is a common notation that the item $m$ in the domain of a man's preference order and the item $w$ in the domain of a woman's preference order stand for remaining single.

11 For a comprehensive discussion of marriage markets, see Roth and Sotomayor (1990, Chapter 2).
} 
McVitie and Wilson (1971) implies that the outcome of the DAB algorithm is not affected by our assumption that the central planner assigns the coordinated agents first; in the DAB search process, the output matching would be the same even if the $\mathrm{CP}$ would assign the controlled agents when the free agents were already searching in the market. This is true as long as the coordinated agents could take away any task already occupied by a free agent, an assumption we consider reasonable for those applications we described in Section 2.

We define a coordinated assignment to be a matching $\mu_{C} \subseteq C \times T$ (no free player $f$ is a member of any pair in $\mu_{C}$ ). We denote by $\left(F, T, \succ_{\boldsymbol{F}}\right)_{\mu_{C}}$ a marriage market formed by free agents and those tasks which are not matched under $\mu_{C}$. Formally,

$(F, T, \succ)_{\mu_{C}}=\left(F, T \backslash\left\{t \mid(c, t) \in \mu_{C}\right\}, \succ\right)$.

Here $\succ$ is a preference profile which assigns to each $t \in T$ a linear order $\succ_{t}$ according to (3.1) and to each free agent the order $\succ_{\boldsymbol{f}}$. Given this, the set $\boldsymbol{\mu}^{\text {SAAP }}$ consists of the following assignments:

Definition 1. An assignment $\mu$ is SAAP-feasible for a semiautonomous assignment problem $\left(C \cup F, T, v, \succ_{F}\right)$ if $\mu=\mu_{F} \cup \mu_{C}$ and the matching $\mu_{F}$ is the outcome of the DAB in the market $\left(F, T, \succ_{\boldsymbol{F}}\right)_{\mu_{C}}$.

\section{Solution}

In this section, we first state the problem as a mathematical program. We then transform the program so that it becomes computationally manageable. Let binary variables $x_{i j}$ indicate whether a controlled agent $i \in C$ is assigned to task $j \in T$, i.e. if $x_{i j}=1$, then $i$ is assigned to $j$, and if $x_{i j}=0$, then this is not the case. Likewise, variables $y_{i j}$ indicated whether a free agent $i$ is matched to task $j$. $\boldsymbol{x}$ and $\boldsymbol{y}$ are matrices with $|C|$ rows and $|F|$ rows, respectively. Both of them have $|T|$ columns and their elements are either 0 or 1.

Theorem 1. The solution to the Semi-Autonomous Assignment problem

$\left(C \cup F, T, v, \succ_{\boldsymbol{F}}\right)$,

coincides with the solution to the optimisation problem ${ }^{12}$

$$
\begin{array}{ll}
\max _{\boldsymbol{x}} & \sum_{(i, j) \in(C \times T)} v_{i j} x_{i j}+g(\boldsymbol{x}), \\
\text { s.t. } & \sum_{j \in T} x_{i j} \leq 1 \quad \forall i \in C \\
& \sum_{i \in A} x_{i j} \leq 1 \quad \forall j \in T \\
& x_{i j} \in\{0,1\} \quad \forall(i, j) \in(C \times T)
\end{array}
$$

where $g(\boldsymbol{x})$ is the value of the allocation of free agents to the tasks not assigned to controlled agents by $\boldsymbol{x}$, i.e.

$$
\begin{array}{ll}
g(\boldsymbol{x})=\min _{\boldsymbol{y}} & \sum_{(i, j) \in(F \times T)} v_{i j} y_{i j} \\
\text { s.t. } & \sum_{j \in T} y_{i j} \leq 1 \quad \forall i \in F \\
& \sum_{i \in F} y_{i j} \leq 1-\sum_{i \in C} x_{i j} \quad \forall j \in T \\
& y_{i j}+\sum_{k \succ_{i} j} y_{i k}+\sum_{l \succ_{j} i} y_{l j} \geq 1 \quad \forall(i, j) \in(F \times T) \\
& y_{i j} \in\{0,1\} \quad \forall(i, j) \in(F \times T) .
\end{array}
$$

\footnotetext{
12 In the optimisation problems we use the notation $v_{i j}$ instead of $v(i, j)$ to denote the value of the assignment of agent $i$ to task $j$.
}

Proof. First of all, we show that the solution of the mathematical program above corresponds to a matching. Constraint (4.2) requires each controlled agent to be assigned at most one task and constraint (4.3) requires each task to be allocated to at most one controlled agent. Likewise, constraint (4.6) requires each free agent to be assigned at most one task and constraint (4.7) requires a controlled task to be allocated to no free agent if it is already occupied by a controlled agent, or to at most one free agent otherwise. Constraints (4.4) and (4.9) ensure that all variables $x_{i j}$ and $y_{i j}$ are binary.

Next, we show that the matrix $\boldsymbol{y}$ determined in the solution of the program corresponds to the outcome of the DAB procedure when only those tasks are available which are not occupied by controlled agents. As mentioned, (4.7) ensures that no free agent is matched to a task assigned to a controlled agent. As we showed in Section 3, the DAB procedure converges to a stable matching in a marriage market $\left(F, T, \succ_{\boldsymbol{F}}\right)_{\mu_{C}}$ (cf. (3.2) above). We now have to show that the matrix $\boldsymbol{y}$ in a solution of the program above corresponds (1) to a stable matching in the market $\left(F, T, \succ_{\boldsymbol{F}}\right)_{\mu_{C}}$, where $\mu_{C}$ is determined by the matrix $\boldsymbol{x}$, and (2) that this stable matching corresponds to that stable matching chosen by the free agents in the DAB search process.

For ensuring that $\boldsymbol{y}$ corresponds to a stable matching in the market $\left(F, T, \succ_{\boldsymbol{F}}\right)_{\mu_{C}}$, we include the so-called blocking pair constraint (4.8). This constraint is taken from Roth et al. (1993), who develop stable matching theory in a mathematical programming framework. If (4.8) is fulfilled, there can be no blocking pairs.

Finally, we have to show that the matching $\boldsymbol{y}$ derived from the solution of the above program is not just stable, but it is indeed the same stable matching as the one constructed through the $D A B$ procedure. In $D A B$, free agents represent the proposing side, and the procedure converges to a stable matching that is optimal for the free agents: each free agent prefers this stable matching to any other stable matching (see Gale and Shapley, 1962). The optimal stable matching for the proposing side coincides with the worst stable matching of the responding side (Roth and Sotomayor, 1990, Theorem 2.13 and Corollary 2.14, p. 33), which means that each task that is not occupied by a controlled agent prefers any other stable matching in the market $\left(F, T, \succ_{\boldsymbol{F}}\right)_{\mu_{C}}$ over the matching selected through DAB. Let $t$ be a task which is not occupied by a controlled agent and let $\left(t, f^{*}\right)$ be the pair formed under the matching constructed through the DAB algorithm. Moreover, let $B(t) \subseteq T \times F$ be the set

$B(t)$

$$
:=\left\{(t, f) \mid(t, f) \subseteq \mu, \mu \text { is a stable matching in }\left(F, T, \succ_{\boldsymbol{F}}\right)_{\mu_{C}}\right\} .
$$

By definition of the "preferences" of the tasks (see (3.1) above) the fact that the constructed matching is the task-worst implies that

$$
\left(t, f^{*}\right)=\underset{(t, f) \in B(t)}{\arg \min } v(t, f) .
$$

Put differently, if $F_{t}$ denotes the set of free agents that perform task $t$ in some stable matching in the market $\left(F, T, \succ_{\boldsymbol{F}}\right)_{\mu_{C}}$, then, in the free-agent-optimal matching, task $t$ is performed by the leastqualified among these agents-arg $\min _{f \in F_{t}} v(t, f)$.

It follows that the objective function for the assignment of free agents, which corresponds to the matching chosen by the DAB algorithm in the market $\left(F, T, \succ_{F}\right)_{\mu_{C}}$, is given by (4.5).

The integrality constraint (4.9) can be relaxed as has been shown by Vande Vate (1989, Theorem 16), allowing to replace it with a nonnegativity constraint. 
The SAAP can thus be fully specified as the following bilevel mixed integer linear program SAAP(2LMILP):

$$
\begin{array}{ll}
\max _{\boldsymbol{x}} & \sum_{(i, j) \in(C \times T)} v_{i j} x_{i j}+\sum_{(i, j) \in(F \times T)} v_{i j} y_{i j} \\
\text { s.t. } & \sum_{j \in T} x_{i j} \leq 1 \quad \forall i \in C \\
& \sum_{i \in C} x_{i j} \leq 1 \quad \forall j \in T \\
& x_{i j} \in\{0,1\} \quad \forall(i, j) \in(C \times T) \\
& \text { solves min } \sum_{(i, j) \in(F \times T)} v_{i j} y_{i j} \\
& \sum_{j \in T} y_{i j} \leq 1 \quad \forall i \in F \\
& \sum_{i \in F} y_{i j} \leq 1-\sum_{i \in C} x_{i j} \quad \forall j \in T \\
y_{i j}+\sum_{k \succ_{i} j} y_{i k}+\sum_{l \succ_{j} i} y_{l j} \geq 1 \quad \forall(i, j) \in(F \times T) \\
y_{i j} \geq 0 \quad \forall(i, j) \in(F \times T) .
\end{array}
$$

Formally, we have a mixed integer bilevel optimisation problem-a hierarchical program in which the set of constraints contains a parametric optimisation problem. Solving bilevel programs is difficult in general, let alone solving one with binary variables, and applying known algorithms to the program at hand would yield solutions only for extremely small problem instances. The most popular method for solving bilevel programs is to replace the second level with a set of Karush-Kuhn-Tucker optimality conditions and then add these constraints to the first level to form a Mathematical Program with Equilibrium Constraints (MPEC) (Luo et al., 1996). However, this introduces a set of complementary constraints that are difficult to deal with. ${ }^{13}$ In fact, solving a linear bilevel program in which all functions are linear is already strongly NP-hard (Marcotte and Savard, 2005). ${ }^{14}$ In our case, the upper level contains binary variables and hence the problem is even more difficult.

For devising a way how to practically solve the SAAP problem, we will show that (SAAP(2LMILP)) is equivalent to a disjoint bilinear program, which is much more manageable computationally, ${ }^{15}$ as stated in the following theorem:

Theorem 2. The SAAP bilevel mixed integer linear programming model (SAAP(2LMILP)) is equivalent to the following disjoint bilinear program SAAP(DBL):

$$
\begin{array}{ll}
\max _{\boldsymbol{x}, \lambda, \boldsymbol{\beta}, \boldsymbol{\gamma}} & \sum_{i \in C, j \in T} v_{i j} x_{i j}+\sum_{i \in F} \lambda_{i}+\sum_{j \in T} \beta_{j}\left(1-\sum_{i \in C} x_{i j}\right)+\sum_{(i, j) \in(F \times T)} \gamma_{i j} \\
\text { s.t. } & \sum_{j \in T} x_{i j} \leq 1 \quad \forall i \in C \\
& \sum_{i \in C} x_{i j} \leq 1 \quad \forall j \in T \\
& x_{i j} \geq 0 \quad \forall(i, j) \in(C \times T) \\
& \lambda_{i}+\beta_{j}+\gamma_{i j}+\sum_{k \prec_{i} j} \gamma_{i k}+\sum_{l \prec_{j} i} \gamma_{l j} \leq v_{i j} \quad \forall(i, j) \in(F \times T) \\
& \lambda \leq 0, \quad \boldsymbol{\beta} \leq 0, \quad \gamma \geq 0 .
\end{array}
$$

\footnotetext{
13 The complementary constraints can then be transformed into a new set of constraints that involve integer variables using a Big-M method. Alternatively, nonlinear programming relaxation can be used to approximate these complementary constraints.

14 Even checking local optimality in linear bilevel programming is NP-hard, cf. Marcotte and Savard (2005).

15 Although solving bilinear programs is still NP-hard (Audet et al., 1999), the mathematical programming formulation is in a much nicer form, i.e. we only have to deal with a single minimisation problem instead of a minimax problem. Notice that not all linear bilevel programs can be transformed into a bilinear problem. However, a disjoint bilinear program can be transformed into a linear bilevel program.
}

At a high level, the transformation of (SAAP(2LMILP)) into a bilinear program involves three steps. First, we replace the linear program on the second level with its dual. Since the primal was a minimisation problem, the dual is a maximisation problem. Having maximisation in both first and second stages lets us combine the objectives and reduce the problem to a single-stage optimisation. The resulting problem belongs to the class of mixed integer nonconvex quadratic programming problems and is still quite difficult to solve. We then exploit the special structure of the problem to note that the integrality constraints on $\boldsymbol{x}$ can be dropped obtaining a bilinear program. The details follow in the proof.

Proof. Let $\lambda_{i}, \beta_{j}$ and $\gamma_{i j}$ be dual variables for constraints (4.6)-(4.8) for all $(i, j) \in(F \times T)$. The dual problem is formulated as:

$$
\begin{array}{ll}
\max _{\lambda, \boldsymbol{\beta}, \boldsymbol{\gamma}} & \sum_{i \in F} \lambda_{i}+\sum_{j \in T}\left(1-\sum_{i \in C} x_{i j}\right) \beta_{j}+\sum_{(i, j) \in(F \times T)} \gamma_{i j} \\
\text { s.t. } & \lambda_{i}+\beta_{j}+\gamma_{i j}+\sum_{k \prec_{i} j} \gamma_{i k}+\sum_{l \prec_{j} i} \gamma_{l j} \leq v_{i j} \quad \forall(i, j) \in(F \times T) \\
& \lambda \leq 0, \quad \boldsymbol{\beta} \leq 0, \quad \boldsymbol{\gamma} \geq 0 .
\end{array}
$$

Plugging the dual into the original problem and combining two max operators, we obtain the following problem:

$$
\begin{array}{ll}
\max _{\boldsymbol{x}, \boldsymbol{\lambda}, \boldsymbol{\beta}, \boldsymbol{\gamma}} & \sum_{i \in C, j \in T} v_{i j} x_{i j}+\sum_{i \in F} \lambda_{i}+\sum_{j \in T} \beta_{j}\left(1-\sum_{i \in C} x_{i j}\right)+\sum_{(i, j) \in(F \times T)} \gamma_{i j} \\
\text { s.t. } & \sum_{j \in T} x_{i j} \leq 1 \quad \forall i \in C \\
& \sum_{i \in C} x_{i j} \leq 1 \quad \forall j \in T \\
& x_{i j} \in\{0,1\} \quad \forall(i, j) \in(C \times T) \\
& \lambda_{i}+\beta_{j}+\gamma_{i j}+\sum_{k \succ_{i} j} \gamma_{i k}+\sum_{l \succ_{j} i} \gamma_{l j} \leq v_{i j} \quad \forall(i, j) \in(F \times T) \\
& \lambda \leq 0, \quad \boldsymbol{\beta} \leq 0, \quad \boldsymbol{\gamma} \geq 0 .
\end{array}
$$

The objective function contains linear terms on $(\boldsymbol{x}, \boldsymbol{\lambda}, \boldsymbol{\beta}, \boldsymbol{\gamma})$ and a bilinear term $-\left(\sum_{(i, j) \in(C \times T)} \beta_{j} x_{i j}\right)$. Without this bilinear term, the problem will be equivalent to two separate optimisation problems: an assignment problem and a (dual of a) stable matching problem. Due to the presence of the bilinear terms together with the integrality constraint on $x_{i j}$, this problem belongs to the class of mixed integer non-convex quadratic programming problems and is quite difficult to solve. However, once we fix $(\boldsymbol{\lambda}, \boldsymbol{\beta}, \boldsymbol{\gamma})$, the objective function is linear on $\boldsymbol{x}$. The problem becomes:

$$
\begin{array}{ll}
\max _{\boldsymbol{x}} & \sum_{i \in C, j \in T}\left(v_{i j}-\beta_{j}\right) x_{i j} \\
\text { s.t. } & \sum_{j} x_{i j} \leq 1 \quad \forall i \in C \\
& \sum_{i \in C} x_{i j} \leq 1 \quad \forall j \in T \\
& x_{i j} \in\{0,1\} .
\end{array}
$$

This is an assignment problem, ${ }^{16}$ and hence the integrality constraint can be relaxed (see, for example, Bertsimas and Tsitsiklis, 1997 , Corollary 7.2). Thus, for every solution $\boldsymbol{\lambda}, \boldsymbol{\beta}, \boldsymbol{\gamma}$ (or equivalently, for every $\boldsymbol{y}$ ) to the agent-optimal stable matching problem, there is an integer solution $\boldsymbol{x}$ that is optimal. In other

\footnotetext{
16 In the variant of the assignment problem stated here, the number of tasks may be different from the number of agents and tasks/agents may be left unassigned. One can convert this version to the standard assignment problem by adding dummy tasks/agents.
} 
words, there is always an integer solution $\boldsymbol{x}$ that is optimal, and we can drop the integrality constraints in SAAP. This leads to the disjoint bilevel program SAAP(DBL).

There is an extensive literature on bilinear programming. In particular, a simple greedy approach, such as 'hill climbing' (see, e.g., Russell and Norvig, 2003), may obtain high-quality solutions in little time. This is done by iteratively solving an LP for optimal $(\boldsymbol{\lambda}, \boldsymbol{\beta}, \boldsymbol{\gamma})$ for each fixed $\boldsymbol{x}$ and then solving another LP for an optimal $\boldsymbol{x}$ by fixing the newly found $(\boldsymbol{\lambda}, \boldsymbol{\beta}, \boldsymbol{\gamma})$. This process is repeated until the optimal value does not improve. At that point we obtain a locally optimal solution. It is noted also that the disjoint constraint sets in the SAAP problem are quite nice. The constraints on $\boldsymbol{x}$ define an assignment polyhedron with known extreme points and the constraints on $(\boldsymbol{\lambda}, \boldsymbol{\beta}, \boldsymbol{\gamma})$ correspond to a dual feasible space of the stable matching problem. This means the LP problems can be solved very efficiently and the algorithm converges very fast to a local optimal solution. White (1992) converts a bilinear program into a big LP whose constraints are generated sequentially through solving smaller LPs. This methods promises finite convergence and can be used to solve SAAP(DBL) as the assignment problem and the stable matching problem can be solved very efficiently. The bilinear program can also be reduced to a concave minimisation problem where an outer approximation algorithm can be applied (Thieu, 1988). More recent advanced methods for solving disjoint bilinear programming can be found in Alarie et al. (2001) who apply cutting plane methods to produce global optimal solutions. Alarie et al. (2001) show that cutting plane methods can be used to solve disjoint bilinear programming problems with up to 500 variables in each disjoint set and with 100 constraints.

\section{Conclusions}

Our work introduces assignment problems in which autonomous agents are placed together with those fully controlled by a central planner. The autonomous agents act to obtain their own individual goals. The central planner coordinates the controlled agents with the aim to optimise the overall performance of the system, while taking into account the behaviour of the self-motivated participants. This scenario resembles many economic situations, some of which were outlined in Section 2.

Clearly, the search process assumed for the free agents in SAAP is not the only reasonable model. Indeed, there are many other possibilities for how one could model the behaviour of the free agents. For example, many real-world scenarios could be better described with a stochastic search process. One might also consider search strategies taken from cognitive psychology, like the famous satisficing heuristic of Simon (1957) or the take-the-best heuristic of Gigerenzer and Goldstein (1996). It may be a worthwhile effort to perform a similar analysis like the one presented in this paper, but with alternative behavioural assumptions for the free agents.

Despite of its various reasonable alternatives, we want to stress that the search process modelled in this article has some intriguing features. Firstly, it is quite natural to assume that the free agents check for free tasks according to some linear order. Secondly, the outcome assignment does not hinge on the assumption that controlled agents are assigned first. This follows from McVitie and Wilson (1971), as discussed in Section 3. Thirdly, in the deferred acceptance algorithm of Gale and Shapley (1962) there is no incentive for the proposing side, in our case the free agents, to misrepresent their preferences (cf. Dubins and Freedman, 1981 and Roth, 1982). In our context, this means that the free agents cannot improve their outcome by changing the order in which they approach tasks. So, even if free agents would have enough information and computing power to act strategically, it would not be worthwhile doing so. In contrast, alternative models of search behaviour would have to take care of strategic manipulations on the free agents' parts. Admittedly, the latter two points make handling our model merely convenient, while they provide no support for the empirical validity of the DAB assumption.

Other modifications to our model come to mind. It may be interesting to change the informational assumptions of the model. What if the productivities of the autonomous workers for different tasks is private knowledge of that worker? ${ }^{17}$ Would there be a way to make the free agents reveal their private information? Could they even be incentivised to pick the task which would be best from the central planner's point of view? Designing a transfer scheme to achieve such goals would demand the free agents to be modelled with cardinal preferences. Arguably, this would reduce the robustness of the model, but it might lead to economically interesting dynamics similar to those which can be found in the famous labour market adjustment models of Crawford and Knoer (1981) and Kelso and Crawford (1982).

The idea of introducing autonomous agents in scenarios where the central planner normally has full control is not limited to assignment problems. Many other standard problems could be extended to include autonomous agents. Transportation or network flow with some transfers performed by autonomous agents, knapsack where autonomous agents are able to add their own items to the knapsack, and graph colouring with some nodes coloured by the agents are just a few examples.

\section{References}

Alarie, S., Audet, C., Jaumard, B., Savard, G., 2001. Concavity cuts for disjoint bilinear programming. Math. Program. 90, 373-398.

Audet, C., Hansen, P., Jaumard, B., Savard, G., 1999. A symmetrical linear maxmin approach to disjoint bilinear programming. Math. Program. 85, 573-592.

Benkler, Y., 2006. The Wealth of Networks: How Social Production Transforms Markets and Freedom. Yale University Press.

Bertsimas, D., Tsitsiklis, J., 1997. Introduction to Linear Optimization. Athena Scientific, Belmont, MA

Brabham, D.C., 2008. Crowdsourcing as a model for problem solving. Convergence $14,75-90$

Crawford, V.P., Knoer, E.M., 1981. Job matching with heterogeneous firms and workers. Econometrica 49, 437-450

Dubins, L.E., Freedman, D.A., 1981. Machiavelli and the Gale-Shapley algorithm. Amer. Math. Monthly 88, 485-494.

Gale, D., Shapley, L.S., 1962. College admissions and the stability of marriage. Amer. Math. Monthly 69, 9-15.

Gigerenzer, G., Goldstein, D.G., 1996. Reasoning the fast and frugal way: models of bounded rationality. Psychol. Rev. 103, 650-669.

Howe, J., 2008. Crowdsourcing: Why the Power of the Crowd is Driving the Future of Business. Crown Business.

Kelso, A.S., Crawford, V.P., 1982. Job matching, coalition formation, and gross substitutes. Econometrica 50, 1483-1504.

Koopmans, T.C., Beckmann, M., 1957. Assignment problems and the location of economic activities. Econometrica 25, 53-76

Luo, Z., Pang, J., Ralph, D., 1996. Mathematical Programs with Equilibrium Constraints. Cambridge University Press.

Marcotte, Patrice, Savard, Gilles, 2005. Bilevel programming: a combinatorial perspective. In: Avis, David, Hertz, Alain, Marcotte, Odile (Eds.), Graph Theory and Combinatorial Optimization. Springer, US, pp. 191-217.

McVitie, D.G., Wilson, L.B., 1971. The stable marriage problem. Commun. ACM 14, 486-492.

Nikolic, I.A., Maikisch, H., 2006. Public-private partnerships and collaboration in the health sector. HNP Discussion Paper (http://info.worldbank.org/etools/docs/ library/240103/PUBLIC 2 .PDF).

Riis, J.A., 2007. Hero Tales of the Far North. Echo Library.

Roth, A.E., 1982. The economics of matching: stability and incentives. Math. Oper. Res. 7, 617-628.

Roth, A., Rothblum, U., Vande Vate, J., 1993. Stable matchings, optimal assignments, and linear programming. Math. Oper. Res. 18, 803-828.

Roth, A.E., Sotomayor, M., 1990. Two-Sided Matching-A Study in Game Theoretic Modeling and Analysis. Cambridge University Press.

Russell, S., Norvig, P., 2003. Artificial Intelligence: A Modern Approach, second ed. Prentice-Hall, Englewood Cliffs, NJ.

Simon, H.A., 1957. Models of Man: Social and Rational. Wiley.

Templeton, S.K., 2007. Dentists refuse to treat bad Teeth. The Sunday Times.

Thieu, T., 1988. A note on the solution of bilinear programming problems by reduction to concave minimization. Math. Program. 41, 249-260.

Vande Vate, J., 1989. Linear programming brings marital bliss. Oper. Res. Lett. 8 ,

White, D., 1992. A linear programming approach to solving bilinear programmes. Math. Program. 56, 45-50.

17 We thank Ulrich Pferschy for suggesting this modification. 